\title{
Nonlinear-dissipation-induced entanglement of coupled nonlinear oscillators
}

\author{
Aurora Voje, ${ }^{*}$ Andreas Isacsson, and Alexander Croy ${ }^{\dagger}$ \\ Department of Applied Physics, Chalmers University of Technology, SE-412 96 Göteborg, Sweden
}

(Received 31 May 2013; published 8 August 2013)

\begin{abstract}
The quantum dynamics of two weakly coupled nonlinear oscillators is analytically and numerically investigated in the context of nonlinear dissipation. The latter facilitates the creation and preservation of nonclassical steady states. Starting from a microscopic description of two oscillators individually interacting with their dissipative environments, it is found that in addition to energy relaxation, dephasing arises due to the mutual coupling. Using the negativity as an entanglement measure, it is shown that the coupling entangles the oscillators in the long-time limit. For finite temperatures, entanglement sudden death and rebirth are observed.
\end{abstract}

DOI: 10.1103/PhysRevA.88.022309

PACS number(s): 03.67.Bg, 03.65.Yz, 85.85.+j

\section{INTRODUCTION}

The counterintuitive concept of dissipation-induced generation of quantum states has recently attracted a lot of interest. It has been proposed to create pure states [1] and, in particular, entangled states [2-6].

Entanglement is among the most striking features of quantum mechanics [7] and a prerequisite for quantum computation and simulation schemes [8,9]. Typically, for dissipation to induce entanglement, the dissipative processes require multiquanta exchange with the environment in addition to carefully engineered coupling constants. Realizations of artificial system-environment couplings are, in general, hard to achieve. On the other hand, it has been shown that multiquanta dissipation is a natural component in nonlinear systems [10]. For instance, graphene-based nanomechanical resonators possess a strong intrinsic nonlinearity [11] and exhibit nonlinear dissipation [12]. In other setups the conservative nonlinearity has to be induced by coupling to auxiliary systems [13]. Similarly, nonlinear dissipation can be induced in optomechanical systems [14-16] and has also been proposed to emerge in superconducting solid-state quantum devices [17].

For a single-oscillator mode of a nonlinear system under the influence of two-quanta dissipation, resulting from a nonlinear coupling to the reservoir, it was shown that nonclassical steady states with nonzero coherences can be generated [12,18-21]. Moreover, the formation of superposition states [15,17] as well as nonthermal and squeezed states was shown to be obtainable by two-phonon cooling [14].

Here, we investigate two coupled nonlinear oscillators, which are individually subject to two-quantum dissipation. We start from a microscopic description of the total system and derive a quantum master equation (QME) for the reduced density matrix. By using the rotating-wave approximation (RWA) we show that in the weak-coupling limit, the two-quantum dissipation mainly influences the short-time dynamics. Additionally, dephasing is affecting the system in the long-time limit. By numerically solving the QME and the QME in the RWA, we

\footnotetext{
*aurora@chalmers.se

†croy@chalmers.se
}

demonstrate that at zero temperature entanglement between the oscillators is created, which persists even if the coupling is switched off. For sufficiently large temperatures, we find earlystage disentanglement (ESD), also known as sudden death of entanglement [22].

\section{MODEL}

We consider two nonlinear oscillators, which are linearly coupled. Additionally, each oscillator is quadratically coupled to a reservoir consisting of harmonic oscillators. Accordingly, the total Hamiltonian is $H=H_{\mathrm{S}}+H_{\mathrm{B}}+H_{\mathrm{SB}}$, where $\hbar=1$, the masses of the oscillators are $m=1$, and

$$
\begin{aligned}
H_{\mathrm{S}} & =\sum_{m=1,2}\left(\frac{1}{2} p_{m}^{2}+\frac{1}{2} \omega_{m}^{2} q_{m}^{2}+\frac{2 \mu_{m}}{3} q_{m}^{4}\right)+\sqrt{\omega_{1} \omega_{2}} \lambda q_{1} q_{2}, \\
H_{\mathrm{B}} & =\sum_{m} \sum_{k} \omega_{m k} b_{m k}^{\dagger} b_{m k}, \\
H_{\mathrm{SB}} & =\sum_{m} q_{m}^{2} \sum_{k}\left(2 \omega_{m} \eta_{m k}\right)\left(b_{m k}^{\dagger}+b_{m k}\right) .
\end{aligned}
$$

Here, $p_{m}=i \sqrt{\omega_{m} / 2}\left(a_{m}^{\dagger}-a_{m}\right)$ and $q_{m}=\left(a_{m}^{\dagger}+a_{m}\right) / \sqrt{\left(2 \omega_{m}\right)}$ denote the momentum and oscillation amplitude of oscillator $m$, respectively, and $a_{m}^{(\dagger)}$ is the annihilation (creation) operator of the $m$ th oscillator. The oscillators are characterized by their frequencies $\omega_{m}$, the strength of the nonlinearities $\mu_{m}$, and the coupling strength $\lambda>0$. The operator $b_{m k}^{\dagger}\left(b_{m k}\right)$ creates (destroys) a phonon in state $k$ of reservoir $m$ with the frequency $\omega_{m k}$. The coupling strength of oscillator $m$ to reservoir state $k$ is denoted by $\eta_{m k}$. The nonlinear coupling in (1c) will lead to nonlinear damping in the classical limit [10]. For nanomechanical systems such a coupling is realized, for example, by coupling of in-plane and flexural motion [23]; in optomechanical setups [14-16] and in superconducting solid-state quantum devices [17] it can be induced by using an auxiliary system.

In the weak system-reservoir coupling limit, the evolution of the reduced density matrix $\rho$ is given by a QME. Following the standard approach by using the Born-Markov approximation in the interaction picture with respect to $H_{\mathrm{S}}$, 
one obtains [24]

$$
\begin{aligned}
\frac{\partial}{\partial t} \rho(t)= & -\sum_{l, m} \int_{0}^{\infty} d \tau\left[S_{l}(t), S_{m}(t-\tau) \rho(t)\right] C_{l m}(\tau) \\
& -\left[S_{l}(t), \rho(t) S_{m}(t-\tau)\right] C_{m l}(-\tau) .
\end{aligned}
$$

Here, the operators $S_{m}(t)=e^{i H_{\mathrm{S}} t}\left(a_{m}^{\dagger}+a_{m}\right)^{2} e^{-i H_{\mathrm{S}} t}$ and $B_{m}(t)=\sum_{k} \eta_{m k}\left(b_{m k}^{\dagger} e^{i \omega_{m k} t}+b_{m k} e^{-i \omega_{m k} t}\right)$ were used to decompose the coupling Hamiltonian $H_{\mathrm{SB}}(t)=\sum_{m=1,2} S_{m}(t) \otimes$ $B_{m}(t)$ in the interaction picture. Assuming the reservoirs to be initially in thermal equilibrium, $\rho_{\mathrm{B}}=\rho_{\mathrm{B}, 1} \otimes \rho_{\mathrm{B}, 2}$, the reservoir correlation functions $C_{m l}(\tau)=\operatorname{Tr}_{\mathrm{B}}\left\{B_{m}(t) B_{l}(t-\tau) \rho_{\mathrm{B}}\right\}$ are given by

$$
C_{m l}(\tau)=\delta_{m l} \int \frac{d \omega}{2 \pi} \kappa_{m}(\omega)\left\{N(\omega) e^{i \omega \tau}+[N(\omega)+1] e^{-i \omega \tau}\right\},
$$

where $N(\omega)=\left(e^{\omega / k_{\mathrm{B}} T}-1\right)^{-1}$ is the Bose-Einstein distribution and $\kappa_{m}(\omega)=2 \pi \sum_{k}\left|\eta_{m k}\right|^{2} \delta\left(\omega-\omega_{m k}\right)$ is the spectral density. The specific form of $\kappa_{m}$ depends on the microscopic details of the system-reservoir coupling. If $\kappa_{m}$ is sufficiently smooth around the frequencies of interest, the exact frequency dependence is not crucial. To be specific, we use an Ohmic spectral density, $\kappa_{m}(\omega)=\Gamma_{m} \omega /\left(2 \omega_{m}\right)$, where $\Gamma_{m}$ is the nonlinear dissipation strength.

In many systems the time scales associated with $\omega_{m}$ and $\mu_{m}$ are well separated. In this case the RWA can be used to simplify the QME. For convenience we consider a symmetric setup in the following, i.e., $\omega_{1}=\omega_{2}=\omega_{0}$ and $\Gamma_{1}=\Gamma_{2}=\Gamma_{0}$.

Further, we define the one-sided Fourier transform of the reservoir correlation function,

$$
\frac{1}{2} \gamma_{m}(\omega)+i \sigma_{m}(\omega)=\int_{0}^{\infty} d \tau e^{i \omega \tau} C_{m m}(\tau) .
$$

The rates $\gamma_{m}$ determine the strength of dissipation, while $\sigma_{m}$ renormalizes the system Hamiltonian. Using the expression of the bath correlation function (3), one finds that

$$
\begin{aligned}
\gamma_{m}\left(2 \omega_{0}\right) & =\Gamma_{0}\left[N\left(2 \omega_{0}\right)+1\right], \\
\gamma_{m}\left(-2 \omega_{0}\right) & =\Gamma_{0} N\left(2 \omega_{0}\right) .
\end{aligned}
$$

In the weak-coupling limit, $\lambda \ll \omega_{0}$, one finally obtains the QME in RWA,

$$
\begin{aligned}
\frac{\partial}{\partial t} \rho(t)= & -i\left[H_{\mathrm{RWA}}, \rho\right]+\sum_{m}\left\{\gamma_{m}\left(2 \omega_{0}\right) \mathcal{L}\left[a_{m}^{\dagger} a_{m}^{\dagger}\right]\right. \\
& \left.+\gamma_{m}\left(-2 \omega_{0}\right) \mathcal{L}\left[a_{m} a_{m}\right]\right\} \rho+\mathcal{D}_{12}(\lambda) \rho
\end{aligned}
$$

in Schrödinger representation. The oscillator Hamiltonian in RWA is given by

$$
\begin{aligned}
H_{\mathrm{RWA}}= & \sum_{m}\left[\left(\omega_{0}+\mu_{m}\right) a_{m}^{\dagger} a_{m}+\mu_{m} a_{m}^{\dagger} a_{m} a_{m}^{\dagger} a_{m}\right] \\
& +\frac{\lambda}{2}\left(a_{1}^{\dagger} a_{2}+a_{2}^{\dagger} a_{1}\right),
\end{aligned}
$$

and the superoperator $\mathcal{L}$ is defined as

$$
\mathcal{L}[X] \rho=-\frac{1}{2} X X^{\dagger} \rho-\frac{1}{2} \rho X X^{\dagger}+X^{\dagger} \rho X .
$$

Thus, to lowest order in $\lambda$, the oscillators are individually coupled to their respective reservoirs. The other superoperator
$\mathcal{D}_{12}(\lambda)$ becomes

$$
\begin{aligned}
\mathcal{D}_{12}(\lambda) \rho= & \Upsilon_{+} \mathcal{L}\left[\left(n_{1}-n_{2}\right)\right] \rho \\
& -\frac{1}{2} \Upsilon_{-}\left[\left(n_{1}-n_{2}\right)\left(a_{1}^{\dagger} a_{2}-a_{2}^{\dagger} a_{1}\right) \rho\right. \\
& -\left(a_{1}^{\dagger} a_{2}-a_{2}^{\dagger} a_{1}\right) \rho\left(n_{1}-n_{2}\right) \\
& +\rho\left(a_{1}^{\dagger} a_{2}-a_{2}^{\dagger} a_{1}\right)^{\dagger}\left(n_{1}-n_{2}\right) \\
& \left.-\left(n_{1}-n_{2}\right) \rho\left(a_{1}^{\dagger} a_{2}-a_{2}^{\dagger} a_{1}\right)^{\dagger}\right],
\end{aligned}
$$

where $\Upsilon_{ \pm}=\gamma(\lambda) \pm \gamma(-\lambda)$, with $\gamma(\lambda)=\kappa_{0}(\lambda)[N(\lambda)+1]$ and $\gamma(-\lambda)=\kappa_{0}(\lambda) N(\lambda)$.

The terms in the QME (6) which are proportional to $\gamma_{m}\left(2 \omega_{0}\right)$ describe the loss of two quanta into the bath, while the terms which are proportional to $\gamma_{m}\left(-2 \omega_{0}\right)$ give rise to the absorption of two quanta from the bath. For zero temperature only the former processes are present since $N\left(2 \omega_{0}\right)=0$. In contrast to the contributions involving $\gamma_{m}\left( \pm 2 \omega_{0}\right)$, the superoperator $\mathcal{D}_{12}$ contains two dephasing terms, proportional to $\Upsilon_{+}$and $\Upsilon_{-}$, respectively. One sees that these two terms have a different temperature dependence. For $\lambda \ll k_{\mathrm{B}} T, \Upsilon_{+}>\Upsilon_{-}$, while for $\lambda \gg k_{\mathrm{B}} T$ one has $\Upsilon_{+} \approx \Upsilon_{-}$. Note that, if the oscillators were linearly coupled to the reservoirs, the latter dephasing terms would only arise if $\Gamma_{1} \neq \Gamma_{2}[25]$.

\section{RESULTS}

\section{A. Zero temperature}

First, we concentrate on $T=0$, which implies $\gamma_{m}\left(-2 \omega_{0}\right)=$ $\gamma(-\lambda)=0$. We introduce the basis vector $|n, i\rangle=|n\rangle_{1} \otimes|i\rangle_{2}$, which denotes a state with $n$ quanta in oscillator 1 and $i$ quanta in oscillator 2. From Eq. (6) one sees that a density matrix, which involves the states $|0,0\rangle,|0,1\rangle$, and $|1,0\rangle$, will not be affected by the dissipation and the QME does not lead to a coupling to other states. In other words, the steady state will, in general, not be the ground state $|0,0\rangle$, but rather a mix of superpositions of $|0,0\rangle,|0,1\rangle$, and $|1,0\rangle$. This state can be written as

$$
\begin{aligned}
\rho= & P_{00}|0,0\rangle\left\langle 0,0\left|+P_{10}\right| 1,0\right\rangle\left\langle 1,0\left|+P_{01}\right| 0,1\right\rangle\langle 0,1| \\
& +\left(\rho_{00,10}|0,0\rangle\left\langle 1,0\left|+\rho_{00,01}\right| 0,0\right\rangle\langle 0,1|\right. \\
& \left.+\rho_{10,01}|1,0\rangle\langle 0,1|+\text { H.c. }\right),
\end{aligned}
$$

where weights of the respective states are determined by the initial state and evolve according to the Hamiltonian $H_{\text {RWA }}$ and the superoperator $\mathcal{D}_{12}$. For example, the populations $P_{00}$ and $P_{10}+P_{01}$ can be obtained from the sum of the populations of the even $\left(P_{\text {even }}\right)$ and odd $\left(P_{\text {odd }}\right)$ states in the initial density matrix [26], respectively. This is a result of the invariance of the total Hamiltonian to changes of total parity $\left(q_{1} \rightarrow-q_{1}\right.$ and $\left.q_{2} \rightarrow-q_{2}\right)$. A similar behavior is observed for a single oscillator $[12,18]$, where it leads to the formation of a nonclassical state with nonvanishing coherences.

To investigate the dynamics of the coupled oscillators the QME (2) and the QME in RWA (6) are solved numerically. The Hilbert space is truncated after $M=10$ states for each oscillator. Equation (2) is solved in the eigenbasis of the system Hamiltonian, which corresponds to the WangsnessBloch-Redfield method [27]. Initially, the system is prepared in a product state of two coherent states, $\psi(0)=\left|\alpha_{1}\right\rangle \otimes$ $\left|\alpha_{2}\right\rangle$, where $\left|\alpha_{m}\right\rangle=\exp \left(\alpha_{m} a_{m}^{\dagger}-\alpha_{m}^{*} a_{m}\right)|0\rangle_{m}, \alpha_{m}=\sqrt{2} q_{m}(0)$ 

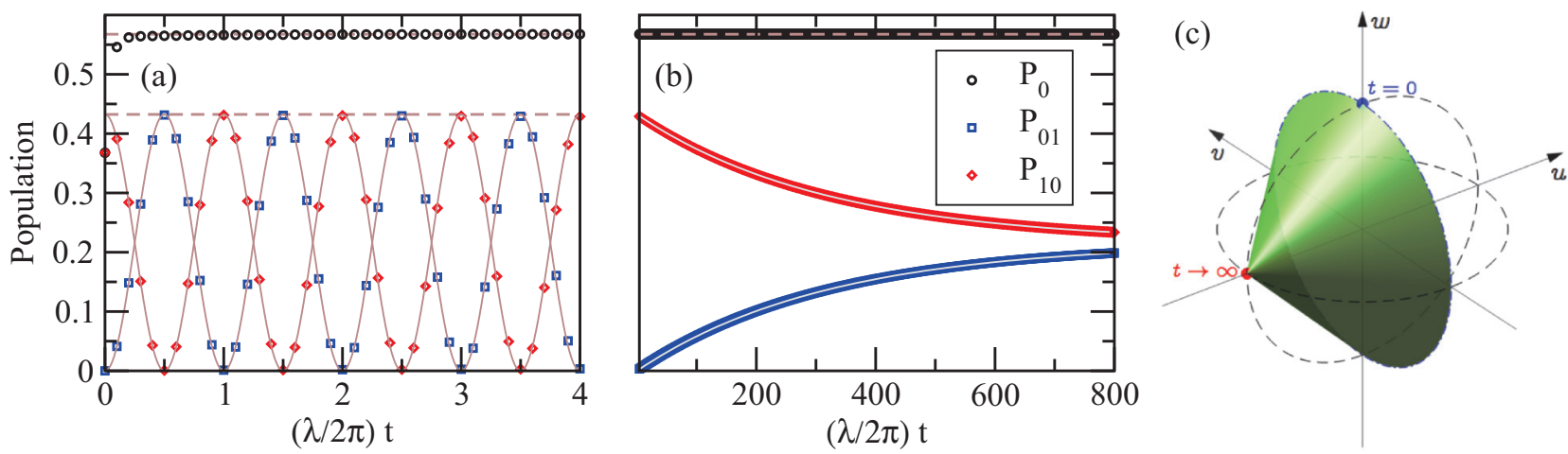

FIG. 1. (Color online) Time dependence of the populations of the states $|0,0\rangle,|1,0\rangle$, and $|0,1\rangle$. The initial oscillator amplitudes are $\alpha_{1}=1$ and $\alpha_{2}=0$. For the calculation $M=10$ states are taken into account for each oscillator. The nonlinear dissipation strength is $\Gamma_{0}=10^{-3}$, the coupling strength between the oscillators is $\lambda=\Gamma_{0} / 2$, and $\mu=\Gamma_{0}$. Thin lines in (a) and (b) are given by Eqs. (11). For visual convenience only the envelope is shown in (b). (c) Dynamics of the Bloch vector $(u, v, w)$ according to Eqs. (11). The cone indicates the states which are attained during the time evolution.

is the amplitude of initial displacement, and the rest of the system parameters are $\omega_{0}=1, \mu_{m}=\mu=\Gamma_{0}=10^{-3}$, and $\lambda=\Gamma_{0} / 2$.

The dynamics of the populations of the states $|0,0\rangle,|1,0\rangle$, and $|0,1\rangle$ is shown in Fig. 1(a) for the situation where only one oscillator is initially displaced ( $\alpha_{1}=1$ and $\left.\alpha_{2}=0\right)$ ). After a transient behavior up to $t \approx 2 \pi / 4 \lambda$, the population of the ground state settles to a constant value $P_{00}=P_{\text {even }}$, and oscillations of $P_{10}$ and $P_{01}$ can be seen, while $P_{01}+P_{10}=P_{\text {odd }}$ remains constant. In the long-time limit, $P_{01}=P_{10}$ due to the presence of dephasing. This is shown in Fig. 1(b). The transient behavior corresponds to the individual relaxation of each oscillator from the initial state to a state which has at most one excitation per oscillator.

Using the QME in RWA (6), the equations of motion for the matrix-elements in the steady state (10) can be solved. It is convenient to introduce $s(t)=P_{01}+P_{10}$ and the components of the Bloch vector $w(t)=P_{10}-P_{01}, u(t)=\rho_{01,10}+\rho_{10,01}$, and $v(t)=-i\left(\rho_{10,01}-\rho_{01,10}\right)$. In the limit $\lambda \gg \Upsilon_{+}=\Upsilon_{-} \equiv$ $\Upsilon$, their solutions are

$$
\begin{aligned}
s(t) & =P_{\text {odd }}, \\
w(t) & =e^{-\Upsilon t}\left[w_{0} \cos (\lambda t)-v_{0} \sin (\lambda t)\right], \\
u(t) & =P_{\text {odd }}\left(e^{-2 \Upsilon t}-1\right)+u_{0} e^{-2 \Upsilon t}, \\
v(t) & =e^{-\Upsilon t}\left[v_{0} \cos (\lambda t)+w_{0} \sin (\lambda t)\right] .
\end{aligned}
$$

In the long-time limit, $w \rightarrow 0$ and $v \rightarrow 0$ while $u \rightarrow-P_{\text {odd }}$, due to dephasing. During its time evolution, the Bloch vector traces the surface of a cone, as can be seen in Fig. 1(c). Without dephasing $(\Upsilon=0)$ it describes a circle.

To quantify the entanglement of the oscillators, which is created by coupling the two systems, the negativity $\mathcal{N}=$ $\left(\left\|\rho^{T_{1}}\right\|_{1}-1\right) / 2$ is computed for each time step [28]. The matrix $\rho^{T_{1}}$ is the partial transpose of the bipartite mixed state $\rho$ with respect to oscillator 1 . The negativity corresponds to the absolute value of the sum of negative eigenvalues of $\rho^{T_{1}}$ and vanishes for any separable state.

For the steady state (10) the negativity is found from the negative roots of the characteristic polynomial of the state's partially transposed density matrix. According to Vieta's formula, the product of the four roots is

$$
z_{1} z_{2} z_{3} z_{4}=-\left|\rho_{01,10}\right|^{2} P_{10} P_{01}
$$

This implies that at least one root $z_{i}$ has to be negative if $P_{10}, P_{01}$, and $\rho_{01,10}$ are nonvanishing, which results in a finite negativity.

The numerically obtained behavior of $\mathcal{N}$ for the case with $\alpha_{1}=1$ and $\alpha_{2}=0$ is shown in Figs. 2(a) and 2(b). An almost periodic behavior is observed, with a vanishing negativity at multiples of a half period $2 \pi / 2 \lambda$. Comparing with Fig. 1(a), one finds that these times correspond to maximal population in either $|1,0\rangle$ or $|0,1\rangle$, which happens twice per period. At those
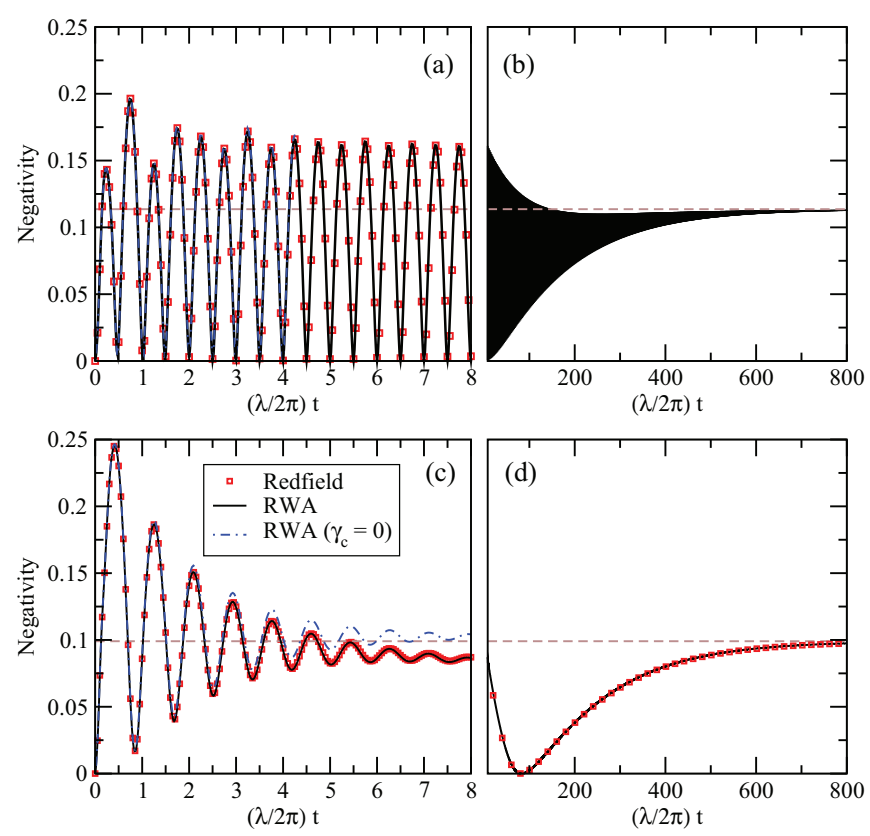

FIG. 2. (Color online) Time dependence of the negativity. The initial oscillator amplitudes are (a) and (b) $\alpha_{1}=1$ and $\alpha_{2}=0$ and (c) and (d) $\alpha_{1}=1$ and $\alpha_{2}=1$. The rest of the parameters are chosen as in Fig. 1. Symbols and solid lines denote numerical results from the Redfield approach and the RWA, respectively. The dashed lines indicate the asymptotic negativity. 
times the coherence between $|1,0\rangle$ and $|0,1\rangle$ vanishes. On the other hand, maximal negativity is found when the coherence is maximal and $P_{10}=P_{01}$, which also happens twice per period. It is important to realize that the entanglement persists even if the coupling is switched off since the steady state (10) is not affected by the nonlinear dissipation, and the dephasing depends on the presence of the coupling $\Upsilon \propto \lambda$.

The time evolution of the negativity is mainly governed by the dynamics of $\left|\rho_{01,10}\right|^{2}=|u / 2|^{2}$. The fast oscillations in Fig. 2(b) can be understood by the circular revolution of the Bloch vector around the $u$ axis in Fig. 1(c). The negativity dip can, in the same manner, be understood in terms of $u(t)$ initially being in the vicinity of the phase space origin in addition to the requirement of Eq. (12) being fulfilled. The negativity saturation is due to $u(t)$ reaching its final value at the tip of the cone as $t \rightarrow \infty$.

In the case of two displaced oscillators, $\alpha_{1}=\alpha_{2}=1$, the populations $P_{00}, P_{01}$, and $P_{10}$ again undergo a short transient behavior and quickly saturate at finite, steady values. The time evolution of the negativity is shown in Figs. 2(c) and 2(d). Initially, the negativity has decaying oscillations, with a period $\approx 2 \pi / \lambda$, which occur due to the influence of decaying coherences between states with more than one excitation. For finite $\Upsilon$, the negativity first decays and then increases to settle at a finite value. This behavior is governed by $\operatorname{Re} \rho_{01,10}=u / 2$, which is initially positive and monotonically decreases according to Eq. (11). Since $w=v=0$, the Bloch vector only points along the $u$ axis. When it crosses the origin, the negativity is minimal. This is also the reason for the absence of long-term oscillations in Fig. 2(d). The asymptotic value of the negativity is given by

$$
\mathcal{N}_{\infty}=\frac{1}{2}\left(P_{\text {odd }}-1+\sqrt{\left(P_{\text {odd }}-1\right)^{2}+P_{\text {odd }}^{2}}\right),
$$

which only depends on the initial state via $P_{\text {odd }}$. If $\Upsilon$ were zero, $u(t)=u(0)$, and the negativity would quickly saturate at a finite value, as seen in Fig. 2(c).

\section{B. Finite temperature}

At finite temperatures the thermal excitations created by the bath will, in general, lead to a decay of the coherences between the Fock states. Therefore, one expects to observe a decay of the entanglement with increasing temperature. In Fig. 3 the time and temperature dependence of the negativity is shown. For temperatures $k_{\mathrm{B}} T / \omega_{0}<1 / 100$ the negativity is slowly decaying with time. For larger temperatures the

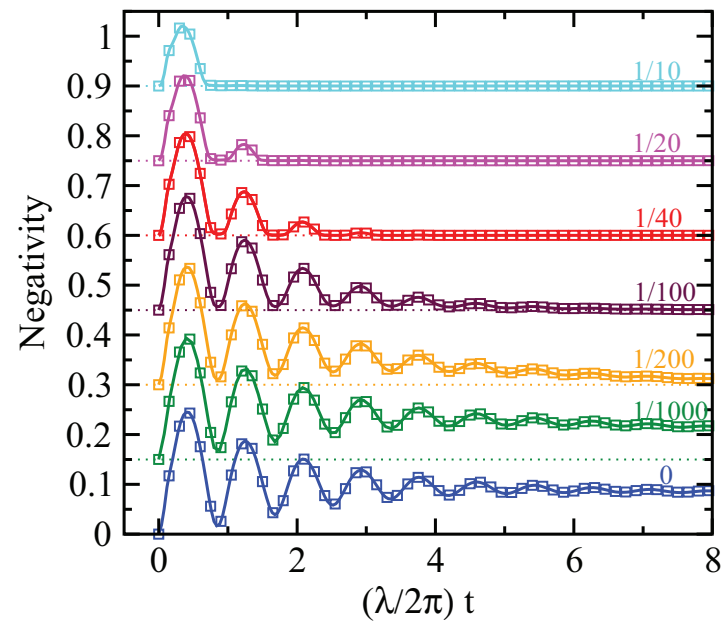

FIG. 3. (Color online) Time and temperature dependence of the negativity. The initial oscillator amplitudes are $\alpha_{1}=\alpha_{2}=1$. The rest of the parameters are chosen as in Fig. 1 . The curves are shifted by 0.15 for convenience. The temperatures in units of $\Omega / k_{\mathrm{B}}$ are given by the labels next to the curves.

negativity is seen to be zero after a finite time. This behavior is known as ESD [22]. These results are also consistent with the previous result [29] that the occurrence of ESD and of entanglement sudden (re)birth at finite temperatures are generic features.

\section{SUMMARY}

In this work we have investigated the quantum dynamics of two weakly interacting anharmonic oscillators, which are nonlinearly coupled to individual dissipative environments. This scenario leads to the formation of nonclassical steady states and, in particular, entanglement. Additionally, at finite temperatures the exotic features of entanglement sudden death and (re)birth are observed. Our results show that dissipationinduced quantum state generation is feasible without engineering the system-environment coupling. Utilizing the natural presence of nonlinear dissipation in nonlinear nanoscale systems provides a promising route to realize exotic quantum state generation.

\section{ACKNOWLEDGMENTS}

The research leading to this article has received funding from the Swedish Research Council (VR).
[1] S. Diehl, A. Micheli, A. Kantian, B. Kraus, H. P. Buchler, and P. Zoller, Nat. Phys. 4, 878 (2008).

[2] F. Verstraete, M. M. Wolf, and J. Ignacio Cirac, Nat. Phys. 5, 633 (2009).

[3] G. Kordas, S. Wimberger, and D. Witthaut, Eur. Phys. Lett. 100, 30007 (2012).

[4] B. Kraus, H. P. Büchler, S. Diehl, A. Kantian, A. Micheli, and P. Zoller, Phys. Rev. A 78, 042307 (2008).

[5] M. J. Kastoryano, F. Reiter, and A. S. Sørensen, Phys. Rev. Lett. 106, 090502 (2011).
[6] H. Krauter, C. A. Muschik, K. Jensen, W. Wasilewski, J. M. Petersen, J. I. Cirac, and E. S. Polzik, Phys. Rev. Lett. 107, 080503 (2011).

[7] E. Schrödinger, Naturwissenschaften 23, 807 (1935).

[8] M. A. Nielsen and I. L. Chuang, Quantum Computation and Quantum Information (Cambridge University Press, Cambridge, 2000).

[9] A. Ekert and R. Jozsa, Philos. Trans. R. Soc. A 356, 1769 (1998).

[10] M. Dykman and M. Krivoglaz, Sov. Sci. Rev. A 5, 265 (1984). 
[11] A. Voje, J. M. Kinaret, and A. Isacsson, Phys. Rev. B 85, 205415 (2012).

[12] A. Voje, A. Croy, and A. Isacsson, New J. Phys. 15, 053041 (2013).

[13] K. Jacobs, Phys. Rev. Lett. 99, 117203 (2007); F. L. Semião, K. Furuya, and G. J. Milburn, Phys. Rev. A 79, 063811 (2009).

[14] A. Nunnenkamp, K. Børkje, J. G. E. Harris, and S. M. Girvin, Phys. Rev. A 82, 021806 (2010).

[15] H. Tan, F. Bariani, G. Li, and P. Meystre, arXiv:1302.7087.

[16] W. Leoński and A. Miranowicz, J. Opt. B 6, S37 (2004).

[17] M. J. Everitt, T. P. Spiller, G. J. Milburn, R. D. Wilson, and A. M. Zagoskin, arXiv:1212.4795.

[18] H. D. Simaan and R. Loudon, J. Phys. A 11, 435 (1978).

[19] L. Gilles and P. L. Knight, Phys. Rev. A 48, 1582 (1993).

[20] L. Gilles, B. M. Garraway, and P. L. Knight, Phys. Rev. A 49, 2785 (1994).

[21] R. Loudon, Opt. Commun. 49, 24 (1984).
[22] T. Yu and J. H. Eberly, Science 323, 598 (2009).

[23] A. Croy, D. Midtvedt, A. Isacsson, and J. M. Kinaret, Phys. Rev. B 86, 235435 (2012).

[24] H. P. Breuer and F. Petruccione, The Theory of Open Quantum Systems (Oxford University Press, New York, 2002).

[25] M. de Ponte, M. de Oliveira, and M. Moussa, Ann. Phys. (NY) 317, 72 (2005).

[26] The definitions are $P_{\text {even }}=\sum_{n, i ; n+i \text { even }} \rho_{n i, n i}$ and $P_{\text {odd }}=$ $\sum_{n, i ; n+i \text { odd }} \rho_{n i, n i}$.

[27] R. K. Wangsness and F. Bloch, Phys. Rev. 89, 728 (1953); F. Bloch, ibid. 105, 1206 (1957); A. G. Redfield, Adv. Magn. Reson. 1, 1 (1965).

[28] G. Vidal and R. F. Werner, Phys. Rev. A 65, 032314 (2002).

[29] A. Al-Qasimi and D. F. V. James, Phys. Rev. A 77, 012117 (2008). 